JOURNAL OF

SYMPLECTIC GEOMETRY

Volume 9, Number 4, 435-444, 2011

\title{
ON THE EXISTENCE OF SYMPLECTIC REALIZATIONS
}

\author{
Marius Crainic and IOAN Mǎrcuț
}

\begin{abstract}
We present a new proof of the existence of symplectic realizations of a Poisson manifold $(M, \pi)$. The proof consists in the construction of a symplectic form on an open neighborhood of the zero-section of the cotangent bundle of $M$, defined by an explicit global formula.
\end{abstract}

\section{Introduction}

Let $(M, \pi)$ be a Poisson manifold. A 'symplectic realization' of $(M, \pi)$ is a symplectic manifold $(S, \omega)$ together with a Poisson submersion

$$
\mu:(S, \omega) \longrightarrow(M, \pi)
$$

Although the existence of symplectic realizations is a fundamental result in Poisson geometry, the known proofs are rather involved. Originally, the local result was proven in $[\mathbf{1 0}]$ and a gluing argument was provided in $[\mathbf{4}]$; the same procedure appears in [7]. The path approach to symplectic groupoids $[\mathbf{1}, \mathbf{3}]$ gives a different proof. Here we present a direct, global, finite dimensional proof, based on the philosophy of 'contravariant geometry': in Poisson geometry, the relevant tangent directions come from the cotangent bundle $T^{*} M$ via the bundle map

$$
\pi^{\sharp}: T^{*} M \longrightarrow T M,
$$

which is just $\pi$ converted into a linear map $\left(\beta\left(\pi^{\sharp}(\alpha)\right)=\pi(\alpha, \beta)\right)$. We will use a contravariant version of the notion of spray.

Definition 0.1. A 'Poisson spray' on a Poisson manifold $(M, \pi)$ is a vector field $\mathcal{V}_{\pi}$ on $T^{*} M$ satisfying the following two properties:

(1) $(d p)_{\xi}\left(\mathcal{V}_{\pi, \xi}\right)=\pi^{\sharp}(\xi)$ for all $\xi \in T^{*} M$,

(2) $m_{t}^{*}\left(\mathcal{V}_{\pi}\right)=t \mathcal{V}_{\pi}$ for all $t>0$,

where $p: T^{*} M \longrightarrow M$ is the canonical projection and $m_{t}: T^{*} M \longrightarrow T^{*} M$ is the fiberwise multiplication by $t>0$. We denote by $\varphi_{t}$ the flow of $\mathcal{V}_{\pi}$. 
A short discussion on Poisson sprays, which are completely analogous to the classical sprays $[\mathbf{8}]$, is given in the next section. Condition (1) means that the integral curves of $\mathcal{V}_{\pi}$ are cotangent paths, it also appears in [11] under the name "second order differential equation".

Our main result is the following.

Theorem 0.1. Given the Poisson manifold $(M, \pi)$ and a contravariant spray $\mathcal{V}_{\pi}$, there exists an open neighborhood $\mathcal{U} \subset T^{*} M$ of the zero-section so that

$$
\omega:=\int_{0}^{1}\left(\varphi_{t}\right)^{*} \omega_{\text {can }} d t
$$

is a symplectic structure on $\mathcal{U}$ and the canonical projection $p:(\mathcal{U}, \omega) \longrightarrow$ $(M, \pi)$ is a symplectic realization.

When $M=U \subset \mathbb{R}^{n}$ open, with $\pi_{p, q}$ the components of $\pi$, the simplest contravariant spray is $\mathcal{V}_{\pi}(x, y)=\sum_{p, q} \pi_{p, q}(x) y_{p} \frac{\partial}{\partial x_{q}}$, where $x$ represents the coordinates of $U$ and $(x, y)$ the induced coordinates on $T^{*} U$. It is not difficult to see that the resulting $\omega$ coincides with the one constructed by Weinstein [10].

One may expect that the proof is "just a computation". Although that is true in principle, the computation is more subtle then one may believe. In particular, we will make use of the principle of "contravariant geometry" which is intrinsic to Poisson geometry. The fact that the proof cannot be so trivial and hides some interesting geometry was already observed in the local case by Weinstein in [10]: the notion of contravariant spray, its existence, and the formula for $\omega$ (giving a symplectic form on an small enough $\mathcal{U}$ ) all make sense for any bivector $\pi$, even if it is not Poisson. But the fact that the pushdown of (the inverse of) $\omega$ is $\pi$ can hold only for Poisson bivectors. Nowadays, with all the insight we have gained from symplectic groupoids, we can say that we have the full geometric understanding of this theorem; in particular, it can be derived from the path-approach to symplectic groupoids of $[\mathbf{1}]$ and the resulting construction of local symplectic groupoids [3]. However, it is clearly worth giving a more direct, global argument.

Let us already start with the first steps of the proof. We will first look at $\omega$ on vectors tangent to $T^{*} M$ at zeros $0_{x} \in T_{x}^{*} M$. At such points one has a canonical isomorphism $T_{0_{x}}\left(T^{*} M\right) \cong T_{x} M \oplus T_{x}^{*} M$ denoted $v \mapsto\left(\bar{v}, \theta_{v}\right)$, and the canonical symplectic form is

$$
\omega_{\text {can }, 0_{x}}(v, w)=\left\langle\theta_{w}, \bar{v}\right\rangle-\left\langle\theta_{v}, \bar{w}\right\rangle .
$$

From the properties of $\mathcal{V}_{\pi}$, it follows that $\varphi_{t}\left(0_{x}\right)=0_{x}$ for all $t$ and all $x$, hence $\varphi_{t}$ is well-defined on a neighborhood of the zero-section, for all $t \in[0,1]$. From the same properties it also follows that

$$
\left(d \varphi_{t}\right)_{0_{x}}: T_{0_{x}}\left(T^{*} M\right) \longrightarrow T_{0_{x}}\left(T^{*} M\right)
$$


is in components given by:

$$
\left(\bar{v}, \theta_{v}\right) \mapsto\left(\bar{v}+t \pi^{\sharp} \theta_{v}, \theta_{v}\right) .
$$

From the definition of $\omega$ and the previous formula for $\omega_{\text {can }}$, we deduce that

$$
\omega_{0_{x}}(v, w)=\left\langle\theta_{w}, \bar{v}\right\rangle-\left\langle\theta_{v}, \bar{w}\right\rangle+\pi\left(\theta_{v}, \theta_{w}\right)
$$

for all $v, w \in T_{0_{x}}\left(T^{*} M\right)$. This implies that $\omega$ is nondegenerate at all zeros $0_{x} \in T^{*} M$. Hence we can find a neighborhood $\mathcal{U}$ of the zero-section in $T^{*} M$ such that $\varphi_{t}$ is defined on $\mathcal{U}$ for all $t \in[0,1]$ and $\left.\omega\right|_{\mathcal{U}}$ is nondegenerate (hence symplectic). Fixing such an $\mathcal{U}$, we still have to show that the map

$$
(d p)_{\xi}: T_{\xi} \mathcal{U} \longrightarrow T_{p(\xi)} M
$$

sends the bivector associated to $\omega$ to $\pi$. The fact that this holds at $\xi=0_{x}$ follows immediately from (2), so our task is to show that it holds at all $\xi \in \mathcal{U}$. Denote by $\mathcal{F}(p) \subset T \mathcal{U}$ the involutive distribution tangent to the fibers of $p$. By Libermann's theorem (see, e.g., Theorem 1.9.7 in [5]), the bivector associated to $\omega$ can be push-down to a bivector on $M$, if and only if $\mathcal{F}(p)^{\perp} \subset \mathcal{U}$, the symplectic orthogonal of $\mathcal{F}(p)$ with respect to $\omega$, is also involutive. What happens in our case is that:

$$
\mathcal{F}(p)^{\perp}=\mathcal{F}\left(p_{1}\right),
$$

where $\mathcal{F}\left(p_{1}\right)$ is the (involutive!) distribution tangent to the fibers of

$$
p_{1}:=p \circ \varphi_{1}: \mathcal{U} \longrightarrow M .
$$

This will be proven in the last section. However, it turns out that the ingredients needed to prove this equality can be used to show directly that $p$ is a Poisson map, without having to appeal to Libermann's result.

\section{Contravariant geometry}

As we have already mentioned, the basic idea of contravariant geometry in Poisson geometry is to replace the tangent bundle $T M$ by the cotangent bundle $T^{*} M$. The two are related by the bundle map $\pi^{\sharp}$. The main structure that makes everything work is the Lie bracket $[\cdot, \cdot]_{\pi}$ on $\Gamma\left(T^{*} M\right)$, which is the contravariant analogue of the Lie bracket on vector fields (the two brackets are related via $\left.\pi^{\sharp}\right)$. It is uniquely determined by the condition

$$
[d f, d g]_{\pi}=d\{f, g\}
$$

and the Leibniz identity

$$
[\alpha, f \beta]_{\pi}=f[\alpha, \beta]_{\pi}+L_{\alpha}(f) \beta
$$

for all $\alpha, \beta \in \Gamma\left(T^{*} M\right)$, where $L_{\alpha}:=L_{\pi^{\sharp} \alpha}$ is the Lie derivative along the ordinary vector field $\pi^{\sharp}(\alpha)$ associated to $\alpha$. In other words, contravariant geometry is the geometry associated to the Lie algebroid $\left(T^{*} M,[\cdot, \cdot]_{\pi}, \pi^{\sharp}\right)$. 
Here are some examples of notions that are contravariant to the usual ones (see e.g. $[\mathbf{3}, \mathbf{6}]$ ).

A 'contravariant connection' on a vector bundle $E$ over $M$ is a a bilinear map

$$
\nabla: \Gamma\left(T^{*} M\right) \times \Gamma(E) \longrightarrow \Gamma(E) \quad(\alpha, s) \mapsto \nabla_{\alpha}(s)
$$

satisfying

$$
\nabla_{f \alpha}(s)=f \nabla_{\alpha}(s), \quad \nabla_{\alpha}(f s)=f \nabla_{\alpha}(s)+L_{\alpha}(f) s
$$

for all $f \in C^{\infty}(M), \alpha \in \Gamma\left(T^{*} M\right), s \in \Gamma(E)$. The standard operations with connections (duals, tensor products, etc.) have an obvious contravariant version.

A 'cotangent path' (or contravariant path) is a path $a:[0,1] \longrightarrow T^{*} M$ sitting above some path $\gamma:[0,1] \longrightarrow M$, such that

$$
\pi^{\sharp}(a(t))=\frac{d \gamma}{d t}(t) .
$$

Intuitively, the cotangent path is the pair $(a, \gamma)$ where $\gamma$ is a standard path and the role of $a$ is to encode "the contravariant derivative of $\gamma$ ". The previous equation says that the contravariant derivative is related to the classical one via $\pi^{\sharp}$.

Given a contravariant connection $\nabla$ on a vector bundle $E$, one has a well-defined notion of 'derivative of sections along cotangent paths': Given a cotangent path $(a, \gamma)$ and a path $u:[0,1] \longrightarrow E$ sitting above $\gamma, \nabla_{a}(u)$ is a new path in $E$ sitting above $\gamma$. Writing $u(t)=s_{t}(\gamma(t))$ for some time dependent section of $E$,

$$
\nabla_{a}(u)=\nabla_{a}\left(s_{t}\right)(x)+\frac{d s_{t}}{d t}(x), \quad \text { at } x=\gamma(t) .
$$

Given a contravariant connection $\nabla$ on $T^{*} M$, the 'contravariant torsion' of $\nabla$ is the tensor $T_{\nabla}$ defined by

$$
T_{\nabla}(\alpha, \beta)=\nabla_{\alpha}(\beta)-\nabla_{\beta}(\alpha)-[\alpha, \beta]_{\pi} .
$$

Given a metric $g$ on $T^{*} M$, one has an associated 'contravariant Levi-Civita connection', which is the unique contravariant metric connection $\nabla^{g}$ on $T^{*} M$ whose contravariant torsion vanishes. The corresponding 'contravariant geodesics' are defined as usual as the (cotangent) curves $a$ satisfying $\nabla_{a} a=0$. They are the integral curves of a vector field $\mathcal{V}_{\pi}^{g}$ on $T^{*} M$, called the 'contravariant geodesic vector field'. In local coordinates $(x, y)$, where $x$ represents the coordinates on $M$ and $y$ the coordinates on the fiber,

$$
\mathcal{V}_{\pi}^{g}(x, y)=\sum_{p, q} \pi_{p, q}(x) y_{p} \frac{\partial}{\partial x_{q}}-\sum_{p, q, r} \Gamma_{p, q}^{r}(x) y_{p} y_{q} \frac{\partial}{\partial y_{r}},
$$


where $\Gamma_{p, q}^{r}(x)$ are the coefficients in $\nabla_{d x_{p}}\left(d x_{q}\right)=\sum_{r} \Gamma_{p, q}^{r}(x) d x_{r}$. Geodesics and the geodesic vector field are actually defined for any contravariant connection $\nabla$ on $T^{*} M$, not necessarily of metric type. For instance, any classical connection $\nabla$ on $T^{*} M$ induces a contravariant connection with $\nabla_{\alpha}:=\nabla_{\pi^{\sharp} \alpha}$ which in general is not of metric type.

The existence of contravariant sprays is now clear:

Lemma 1.1. Any contravariant geodesic vector field is a contravariant spray.

Recall also that (cf. e.g. [3]) any classical connection $\nabla$ induces two contravariant connections, one on $T M$ and one on $T^{*} M$, both denoted by $\bar{\nabla}$ :

$$
\bar{\nabla}_{\alpha}(V)=\pi^{\sharp} \nabla_{V}(\alpha)+\left[\pi^{\sharp}(\alpha), V\right], \bar{\nabla}_{\alpha}(\beta)=\nabla_{\pi^{\sharp} \beta}(\alpha)+[\alpha, \beta]_{\pi} .
$$

The two are related by the following lemma, which follows immediately from the fact that $\pi^{\sharp}$ is a Lie algebra map from $\left(\Gamma\left(T^{*} M\right),[\cdot, \cdot]_{\pi}\right)$ to the Lie algebra of vector fields. Note also that this (and its consequences) is the only place where we use that $\pi$ is Poisson.

Lemma 1.2. For any classical connection $\nabla$, the following identity holds:

$$
\bar{\nabla}_{\alpha}\left(\pi^{\sharp}(\beta)\right)=\pi^{\sharp}\left(\bar{\nabla}_{\alpha}(\beta)\right) .
$$

In the next section, we will be using a torsion-free connection $\nabla$, and this will simplify the computations because of the following lemma:

Lemma 1.3. If $\nabla$ is a torsion-free connection and $a:[0,1] \longrightarrow T^{*} M a$ cotangent path with base path $\gamma$, then for any smooth paths $\theta$ in $T^{*} M$ and $v$ in $T M$, both above $\gamma$, the following identity holds:

$$
\left\langle\bar{\nabla}_{a}(\theta), v\right\rangle+\left\langle\theta, \bar{\nabla}_{a}(v)\right\rangle=\frac{d}{d t}\langle\theta, v\rangle .
$$

Proof. Choose a time-dependent 1-form $A=A(t, x)$ such that $a(t)=$ $A(t, \gamma(t))$ and similarly a time-dependent 1-form $\Theta$ corresponding to $\theta$, and a time-dependent vector field $V$ corresponding to $v$. Applying the definition of the derivatives $\bar{\nabla}_{a}$ along cotangent paths, and then the definition of $\bar{\nabla}$, we find that the left-hand side at time $t$ coincides with the following expression on $(t, x)$ evaluated at $x=\gamma(t)$ :

$$
\left\langle\nabla_{\pi^{\sharp} \Theta}(A)+[A, \Theta]_{\pi}+\frac{d \Theta}{d t}, V\right\rangle+\left\langle\Theta, \pi^{\sharp} \nabla_{V}(A)+\left[\pi^{\sharp} A, V\right]+\frac{d V}{d t}\right\rangle .
$$

For the two terms involving $\nabla$ we find

$$
\begin{aligned}
& \left\langle\nabla_{\pi^{\sharp} \Theta}(A), V\right\rangle+\left\langle\Theta, \pi^{\sharp} \nabla_{V}(A)\right\rangle \\
& \quad=\left\langle\nabla_{\pi^{\sharp} \Theta}(A), V\right\rangle-\left\langle\nabla_{V}(A), \pi^{\sharp} \Theta\right\rangle= \\
& \quad=L_{\pi^{\sharp} \Theta}\langle A, V\rangle-\left\langle A, \nabla_{\pi^{\sharp} \Theta}(V)\right\rangle-L_{V}\left\langle A, \pi^{\sharp} \Theta\right\rangle+\left\langle A, \nabla_{V}\left(\pi^{\sharp} \Theta\right)\right\rangle \\
& \quad=L_{\pi^{\sharp} \Theta}\langle A, V\rangle-L_{V}\left\langle A, \pi^{\sharp} \Theta\right\rangle+\left\langle A,\left[V, \pi^{\sharp} \Theta\right]\right\rangle,
\end{aligned}
$$


where we have used the antisymmetry of $\pi$, and then passed from $\nabla$ on $T^{*} M$ to its dual on $T M$, and used that $\nabla$ is torsion-free. For the term in (5) containing $[\cdot, \cdot]_{\pi}$, using the definition of this bracket we find

$$
\begin{aligned}
& \left\langle[A, \Theta]_{\pi}, V\right\rangle=\left\langle L_{\pi^{\sharp} A}(\Theta)-L_{\pi^{\sharp} \Theta}(A)-d \pi(A, \Theta), V\right\rangle \\
& =L_{\pi^{\sharp} A}\langle\Theta, V\rangle-\left\langle\Theta,\left[\pi^{\sharp} A, V\right]\right\rangle-L_{\pi^{\sharp} \Theta}\langle A, V\rangle+\left\langle A,\left[\pi^{\sharp} \Theta, V\right]\right\rangle-L_{V}(\pi(A, \Theta)) .
\end{aligned}
$$

Plugging the last two expressions into (5) we find

$$
\left\langle\frac{d \Theta}{d t}, V\right\rangle+\left\langle\Theta, \frac{d V}{d t}\right\rangle+L_{\pi^{\sharp} A}\langle\Theta, V\rangle .
$$

As an expression on $(t, x)$, when evaluated at $x=\gamma(t)$, since $\pi^{\sharp} A=\frac{d \gamma}{d t}$, we find precisely the right hand side of the identity in the lemma.

\section{A different formula for $\omega$}

In this section, we give another description of $\omega$. The resulting formula is a generalization of formula (2) from zeros $0_{x}$ to arbitrary $\xi \in T^{*} M$. It will depend on a connection $\nabla$ on $T M$ which is used in order to handle vectors tangent to $T^{*} M$. Hence, from now on, we fix such a connection which we assume to be torsion-free. With respect to $\nabla$, any tangent vector $v \in T_{\xi}\left(T^{*} M\right)$ is determined by the tangent vector induced on $M$ and by its vertical component

$$
\bar{v}=(d p)_{\xi}(v) \in T_{p(\xi)} M, \quad \theta_{v}=v-\operatorname{hor}_{\xi}(\bar{v}) \in T_{p(\xi)}^{*} M .
$$

Of course, when $\xi=0_{x}$, these coincide with the components mentioned in the introduction. The fact that $\nabla$ is torsion-free ensures the following generalization of the formula (1) for $\omega_{\text {can }}$ at arbitrary $\xi$.

Lemma 2.1. If $\nabla$ is torsion-free, then for any $v, w \in T_{\xi}\left(T^{*} M\right)$,

$$
\omega_{\mathrm{can}}(v, w)=\left\langle\theta_{w}, \bar{v}\right\rangle-\left\langle\theta_{v}, \bar{w}\right\rangle .
$$

Proof. Since $\nabla$ is torsion free, it follows that the associated horizontal distribution $H \subset T\left(T^{*} M\right)$ is Lagrangian with respect to $\omega_{\text {can }}$ and then the formula follows.

Denote by $\mathcal{U} \subset T^{*} M$ an open neighborhood of the zero section on which $\omega$ is nondegenerate and $\varphi_{t}$ is defined up to time 1 . To establish the generalization of (2) at $\xi \in \mathcal{U}$, we introduce some notation. Fix $\xi \in \mathcal{U}$ and consider

$$
a:[0,1] \longrightarrow \mathcal{U}, a(t)=\varphi_{t}(\xi),
$$

which, from the properties of $\mathcal{V}_{\pi}$, is a cotangent path. We denote by $\gamma=p \circ a$ its base path. By pushing forward a tangent vector $v_{0} \in T_{\xi} \mathcal{U}$ by $\varphi_{t}$ we obtain a smooth path

$$
t \mapsto v_{t}:=\left(\varphi_{t}\right)_{*}\left(v_{0}\right) \in T_{a(t)} \mathcal{U}
$$


The components of $v$ with respect to $\nabla$ are two paths above $\gamma$, one in $T M$ and one in $T^{*} M$, denoted by $\bar{v}$ and $\theta_{v}$

$$
t \mapsto \bar{v}_{t} \in T_{\gamma(t)} M, \quad t \mapsto \theta_{v_{t}} \in T_{\gamma(t)}^{*} M .
$$

They are related in the following way:

Lemma 2.2. For $a, \bar{v}$ and $\theta_{v}$ as above, we have $\bar{\nabla}_{a} \bar{v}=\pi^{\sharp} \theta_{v}$.

Proof. We start with one remark on derivatives along vector fields. For any smooth path of vectors $V$ tangent to $\mathcal{U}$ along $a, t \mapsto V(t) \in T_{a(t)} \mathcal{U}$, one has the Lie derivative of $V$ along $\mathcal{V}_{\pi}$, again a smooth path of tangent vectors along $a$, defined by

$$
L_{\mathcal{V}_{\pi}}(V)(t)=\left.\frac{d}{d s}\right|_{s=0}\left(d \varphi_{-s}\right)_{a(s+t)}(V(s+t)) \in T_{a(t)} \mathcal{U} .
$$

We have the following two remarks:

(1) For vertical $V$, meaning which comes from a 1-form $\theta_{V}$ on $M$ along $\gamma$, we have that $(d p)\left(L_{\mathcal{V}_{\pi}}(V)\right)=-\pi^{\sharp}\left(\theta_{V}\right)$. This follows immediately from the first property of the spray (e.g. by a local computation).

(2) For horizontal $V,(d p)\left(L_{\mathcal{V}_{\pi}}(V)\right)=\bar{\nabla}_{a}(\bar{V})$, where $\bar{V}=(d p)(V)$ is a tangent vector to $M$ along $\gamma$. To check this, one may assume that $V$ is a global horizontal vector field on $M$, and one has to show that $(d p)_{\eta}\left(L_{\mathcal{V}_{\pi}}(V)\right)=\bar{\nabla}_{\eta}(\bar{V})$ for all $\eta \in T^{*} M$. Again, this follows immediately by a local computation.

Hence, for an arbitrary $V$ (along $a$ ), using its components $\left(\bar{V}, \theta_{V}\right)$,

$$
d p\left(L_{\mathcal{V}_{\pi}}(V)\right)=-\pi^{\sharp}\left(\theta_{V}\right)+\bar{\nabla}_{a}(\bar{V}) .
$$

Finally, note that for $v$ as in $(7), L_{\mathcal{V}_{\pi}}(v)=0$.

We have the following version of (2) at arbitrary $\xi$ in $\mathcal{U}$.

Lemma 2.3. Let $\xi \in \mathcal{U}$ and $v_{0}, w_{0} \in T_{\xi}\left(T^{*} M\right)$. Let $v=v_{t}$ as before and let $\widetilde{\theta}_{v}$ be a path in $T^{*} M$, which is a solution of the differential equation

$$
\bar{\nabla}_{a}\left(\widetilde{\theta}_{v}\right)=\theta_{v} .
$$

Similarly, consider $w=w_{t}$ and $\widetilde{\theta}_{w}$ corresponding to $w_{0}$. Then

$$
\omega\left(v_{0}, w_{0}\right)=\left.\left(\left\langle\widetilde{\theta}_{w}, \bar{v}\right\rangle-\left\langle\widetilde{\theta}_{v}, \bar{w}\right\rangle-\pi\left(\widetilde{\theta}_{v}, \widetilde{\theta}_{w}\right)\right)\right|_{0} ^{1} .
$$

Proof. Since $\nabla$ is torsion-free, Lemma 2.1 implies that

$$
\omega\left(v_{0}, w_{0}\right)=\int_{0}^{1}\left(\left\langle\theta_{w}, \bar{v}\right\rangle-\left\langle\theta_{v}, \bar{w}\right\rangle\right) d t .
$$

Hence, it suffices to show that

$$
\left\langle\theta_{w}, \bar{v}\right\rangle-\left\langle\theta_{v}, \bar{w}\right\rangle=\frac{d}{d t}\left(\left\langle\widetilde{\theta}_{w}, \bar{v}\right\rangle-\left\langle\widetilde{\theta}_{v}, \bar{w}\right\rangle-\pi\left(\widetilde{\theta}_{v}, \widetilde{\theta}_{w}\right)\right) .
$$


Using (8) for $\theta_{v}$ and $\theta_{w}$, followed by Lemma 1.3, we obtain

$$
\left\langle\theta_{w}, \bar{v}\right\rangle-\left\langle\theta_{v}, \bar{w}\right\rangle=\frac{d}{d t}\left(\left\langle\widetilde{\theta}_{w}, \bar{v}\right\rangle-\left\langle\widetilde{\theta}_{v}, \bar{w}\right\rangle\right)-\left\langle\widetilde{\theta}_{w}, \bar{\nabla}_{a}(\bar{v})\right\rangle+\left\langle\widetilde{\theta}_{v}, \bar{\nabla}_{a}(\bar{w})\right\rangle .
$$

The last two terms can be evaluated as follows:

$$
\begin{aligned}
-\left\langle\widetilde{\theta}_{w}, \bar{\nabla}_{a}(\bar{v})\right\rangle+\left\langle\widetilde{\theta}_{v}, \bar{\nabla}_{a}(\bar{w})\right\rangle & =-\left\langle\widetilde{\theta}_{w}, \pi^{\sharp}\left(\theta_{v}\right)\right\rangle+\left\langle\widetilde{\theta}_{v}, \pi^{\sharp}\left(\theta_{w}\right)\right\rangle \\
& =-\left\langle\widetilde{\theta}_{w}, \pi^{\sharp}\left(\bar{\nabla}_{a}\left(\widetilde{\theta}_{v}\right)\right)\right\rangle+\left\langle\widetilde{\theta}_{v}, \pi^{\sharp}\left(\bar{\nabla}_{a}\left(\widetilde{\theta}_{w}\right)\right)\right\rangle \\
& =-\left\langle\widetilde{\theta}_{w}, \bar{\nabla}_{a}\left(\pi^{\sharp}\left(\widetilde{\theta}_{v}\right)\right)\right\rangle-\left\langle\bar{\nabla}_{a}\left(\widetilde{\theta}_{w}\right), \pi^{\sharp}\left(\widetilde{\theta}_{v}\right)\right\rangle \\
& =-\frac{d}{d t}\left\langle\widetilde{\theta}_{w}, \pi^{\sharp}\left(\widetilde{\theta}_{v}\right)\right\rangle=-\frac{d}{d t} \pi\left(\widetilde{\theta}_{v}, \widetilde{\theta}_{w}\right),
\end{aligned}
$$

where we have used Lemma (2.2), equation (8), the fact that $\pi^{\sharp}\left(\bar{\nabla}_{a}\left(\widetilde{\theta}_{v}\right)\right)=$ $\bar{\nabla}_{a}\left(\pi^{\sharp}\left(\widetilde{\theta}_{v}\right)\right)$, the antisymmetry of $\pi^{\sharp}$ and Lemma 1.3 .

\section{The proof of the theorem}

We now return to the proof of Theorem 0.1. We start with the proof of the equality (3) from the introduction. By counting dimensions, it suffices to prove the reverse inclusion. Fix $\xi \in \mathcal{U}$. We have to show that $\omega\left(v_{0}, w_{0}\right)=0$ for all

$$
v_{0} \in \mathcal{F}(p)_{\xi}, \text { and } w_{0} \in \mathcal{F}\left(p_{1}\right)_{\xi} .
$$

Using the notation of the previous section, these conditions are equivalent to $\bar{v}(0)=0$ and $\bar{w}(1)=0$. We remark that (8), as an equation on $\widetilde{\theta}_{v}$, is a linear ordinary differential equation; hence it has solutions defined for all $t \in[0,1]$ satisfying any given initial (or final) condition. Hence one may arrange that $\widetilde{\theta}_{v}(0)=0, \widetilde{\theta}_{w}(1)=0$. Lemma 2.3 immediately implies that $\omega\left(v_{0}, w_{0}\right)=0$.

Finally, we show that $p$ is a Poisson map. We have to show for $\xi \in \mathcal{U}$ arbitrary and $\theta \in T_{x}^{*} M(x=p(\xi))$ that the unique $v_{0} \in T_{\xi} \mathcal{U}$ satisfying

$$
p^{*}(\theta)_{\xi}=i_{v_{0}}(\omega)
$$

also satisfies $(d p)_{\xi}\left(v_{0}\right)=\pi^{\sharp}(\theta)$. From (11), it immediately follows that $v_{0}$ is in $\mathcal{F}(p)^{\perp}$, hence in $\mathcal{F}\left(p_{1}\right)$, therefore $\bar{v}(1)=0$. Next, we evaluate (11) on an arbitrary $w_{0} \in T_{\xi} \mathcal{U}$. We also use the formula for $\omega$ from Lemma 2.3, where $\widetilde{\theta}_{v}$ and $\widetilde{\theta}_{w}$ are chosen so that $\widetilde{\theta}_{v}(1)=0$ and $\widetilde{\theta}_{w}(0)=\eta \in T_{x}^{*} M$ is arbitrary. We find:

$$
\theta(\bar{w}(0))=\left\langle\widetilde{\theta}_{v}(0), \bar{w}(0)\right\rangle+\left\langle\eta, \pi^{\sharp} \widetilde{\theta}_{v}(0)-\bar{v}(0)\right\rangle .
$$

Since this holds for all $w_{0}$ and all $\eta$, we deduce that $\theta=\widetilde{\theta}_{v}(0)$ and $\pi^{\sharp} \widetilde{\theta}_{v}(0)=$ $\bar{v}(0)$. Hence $\pi^{\sharp}(\theta)=\bar{v}(0)=(d p)_{\xi}\left(v_{0}\right)$. 


\section{Some remarks}

Here are some remarks on possible variations. First of all, regarding the notion of contravariant spray, the first condition means that, locally, $\mathcal{V}_{\pi}$ is of the form

$$
\mathcal{V}_{\pi}(x, y)=\sum_{p, q} \pi_{p, q}(x) y_{p} \frac{\partial}{\partial x_{q}}+\sum_{i} \gamma^{i}(x, y) \frac{\partial}{\partial y_{i}}
$$

The second condition means that each $\gamma^{i}(x, y)$ is of the form $\sum_{j, k} \gamma_{j, k}^{i}(x) y_{j} y_{k}$. While the first condition has been heavily used in the paper, the second one was only used to ensure that $\omega$ is well-defined and nondegenerate at elements $0_{x} \in T_{x}^{*} M$.

Another remark is that one can show that $\mathcal{U}$ can be made into a local symplectic groupoid, with source map $p$ and target map $p_{1}$ (see also [7]).

Let us also point out that we used that $\pi$ is Poisson only to prove the compatibility relation (4) which in turn was only used at the end of the proof of Lemma 2.3. However, it is easy to keep track of the extra-terms that show up for general bivectors $\pi$. At the right-hand side of (4), one has to add the term $i_{\alpha \wedge \beta}\left(\chi_{\pi}\right)$ where $\chi_{\pi}=[\pi, \pi]$, and to $(9)$ the term $\int_{0}^{1} \chi_{\pi}\left(a, \widetilde{\theta}_{v}, \widetilde{\theta}_{w}\right) d t$. This is useful for handling various twisted versions. For example, for a $\sigma$-twisted bivector $\pi$ on $M$ (i.e., satisfying $[\pi, \pi]=\pi^{\sharp}(\sigma)$, where $\sigma$ is a given closed 3 -form on $M$, see $[\mathbf{9}]$ ), the interesting (twisted symplectic) 2 -form on $\mathcal{U}$ is the previously defined $\omega$ to which we add the 2 -form $\omega_{\sigma}$ given by (compare with [2]):

$$
\omega_{\sigma}=\int_{0}^{1} \varphi_{t}^{*}\left(i_{\mathcal{V}_{\pi}} p^{*}(\sigma)\right) d t
$$

\section{References}

[1] A. Cattaneo and G. Felder, Poisson sigma models and symplectic groupoids, Quantization of singular symplectic quotients, pp. 61-93, Progress in Mathematics, 198, Birkhauser, Basel, 2001.

[2] A. Cattaneo and P. Xu, Integration of twisted Poisson structures, J. Geom. Phys. 49 (2004) 187-196.

[3] M. Crainic and R.L. Fernandes, Integrability of Poisson brackets, J. Diff. Geom. 66 (2004), 71-137.

[4] A. Coste, P. Dazord and A. Weinstein, Groupoides symplectiques, Publ. Dép. Math. Nouvelle Ser. A 2 (1987), 1-62.

[5] J.P. Dufour and N.T. Zung, Poisson structures and their normal forms, in 'Progress in Mathematics', 242, Birkhauser Verlag, Basel, 2005.

[6] R.L. Fernandes, Connections in Poisson geometry, J. Diff. Geom. 54 (2000), 303-365.

[7] M.V. Karasev, Analogues of objects of the theory of Lie groups for nonlinear Poisson brackets, Math. USSR-Izv. 28 (1987), 497-527. 
[8] S. Lang, Introduction to differentiable manifolds, Interscience Publishers (a division of John Wiley \& Sons, Inc.), New York-London 1962.

[9] P. Severa and A. Weinstein, Poisson geometry with a 3-form background, Progr. Theoret. Phys. Suppl. 144 (2001), 145-154.

[10] A. Weinstein, The local structure of Poisson manifolds, J. Diff. Geom. 18 (1983), 523-557.

[11] A. Weinstein, Lagrangian mechanics and groupoids, Fields Inst. Commun. 7 (1996), 207-231.

Department of Mathematics

UTRECHT UNIVERSITY

3508 TA UTRECHT

The NetherLands

E-mail address: M.Crainic@uu.nl

Department of Mathematics

UTRECHT UNIVERSITY

3508 TA UTRECHT

The Netherlands

E-mail address: I.T.Marcut@uu.nl

Received 12/10/2010, accepted 02/16/2011

This research was supported by the NWO Vidi Project "Poisson topology". 